\title{
A Pilot Proficiency Testing Study for Assessing Cancer Gene Panel using Patient Samples and Next- generation Sequencing in Japan
}

Masato Maekawa ( $\triangle$ mmaekawa@hama-med.ac.jp)

Hamamatsu University School of Medicine

Terumi Taniguchi

Hamamatsu University School of Medicine

Kazuto Nishio

Kindai University

Kazuko Sakai

Kindai University

Kazuyuki Matsushita

Chiba University Hospital

Takayuki Ishige

Chiba University Hospital

Kaname Nakatani

Mie University Hospital

Makoto Ikejiri

Mie University Hospital

Hiroshi Nishihara

Keio University

Kuniko Sunami

National Cancer Centre

Yasushi Yatabe

National Cancer Centre

Kanako Hatanaka

Hokkaido University Hospital

Yutaka Hatanaka

Hokkaido University Hospital

Yoshihiro Yamamoto

Kyoto University Hospital

Keita Fukuyama

Kyoto University Hospital

Shinya Oda 
Kyushu Cancer Center

\section{Kayoko Saito}

Tokyo Women's Medical University

\section{Mamoru Yokomura}

Tokyo Women's Medical University

\section{Yuji Kubo}

RIKEN GENESIS Co.

\section{Hiroko Sato}

RIKEN GENESIS Co.

\section{Yoshinori Tanaka}

RIKEN GENESIS Co.

\section{Misa Fuchioka}

SRL (Japan)

\section{Tadashi Yamasaki}

BML (Japan)

\section{Koichiro Matsuda}

LSI Medience Co.

\section{Kiyotaka Kurachi}

Hamamatsu University School of Medicine

\section{Kazuhiro Funai}

Hamamatsu University School of Medicine

\section{Satoshi Baba}

Hamamatsu University School of Medicine

\section{Moriya Iwaizumi}

Hamamatsu University School of Medicine

\section{Research Article}

Keywords: precision oncology, Cancer Gene Panel, Next-generation Sequencing

Posted Date: September 7th, 2021

DOl: https://doi.org/10.21203/rs.3.rs-877835/v1

License: (c) (1) This work is licensed under a Creative Commons Attribution 4.0 International License. Read Full License 
1 A pilot proficiency testing study for assessing cancer gene panel using patient samples and next-

\section{generation sequencing in Japan}

Masato Maekawa ${ }^{*}$, Terumi Taniguchi ${ }^{1}$, Kazuto Nishio $^{2}$, Kazuko Sakai $^{2}$, Kazuyuki Matsushita ${ }^{3}$, Kaname Nakatani $^{4,5}$, Takayuki Ishige ${ }^{3}$, Makoto Ikejiri ${ }^{4}$, Hiroshi Nishihara ${ }^{6}$, Kuniko Sunami ${ }^{7}$, Yasushi Yatabe ${ }^{8}$, Kanako C Hatanaka9, Yutaka Hatanaka ${ }^{9,10}$, Yoshihiro Yamamoto ${ }^{11}$, Keita Fukuyama ${ }^{11}$, Shinya Oda ${ }^{12}$, Kayoko Saito ${ }^{13}$, Mamoru Yokomura ${ }^{13}$, Yuji Kubo ${ }^{14}$, Hiroko Sato ${ }^{15}$, Yoshinori Tanaka ${ }^{15}$, Misa Fuchioka ${ }^{16}$, Tadashi Yamasaki ${ }^{17}$, Koichiro Matsuda ${ }^{18}$, Kiyotaka Kurachi ${ }^{19}$, Kazuhiro Funai ${ }^{20}$, Satoshi Baba ${ }^{21}$, Moriya Iwaizumi $^{1}$

1 Department of Laboratory Medicine, Hamamatsu University School of Medicine, Hamamatsu, Japan 2 Department of Genome Biology, Kindai University Faculty of Medicine, Sayama, Japan

3 Department of Laboratory Medicine, Chiba University Hospital, Chiba, Japan

4 Department of Clinical Laboratory, Mie University Hospital, Tsu, Japan

5 Iga City General Hospital, Iga, Japan

6 Genomics Unit, Keio Cancer Center, Keio University School of Medicine, Tokyo, Japan

7 Department of Laboratory Medicine, National Cancer Center Hospital, Tokyo, Japan

8 Department of Diagnostic Pathology, National Cancer Center Hospital, Tokyo, Japan

9 Center for Development of Advanced Diagnostics, Hokkaido University Hospital, Sapporo, Japan 10 Research Division of Genome Companion Diagnostics, Hokkaido University Hospital, Sapporo, Japan

11 Department of Clinical Oncology, Kyoto University Hospital, Kyoto, Japan 12 Cancer Genetics Laboratory, Clinical Research Institute, National Hospital Organization, Kyushu Cancer Center, Fukuoka, Japan 
2513 Institute of Medical Genetics, Tokyo Women's Medical Genetics, Tokyo, Japan

2614 Genetic Analysis Department, Tsukiji Registered Clinical Laboratory, RIKEN GENESIS CO., LTD.,

27 National Cancer Center, Tokyo, Japan

2815 Genetic Analysis Department, Kawasaki Registered Clinical Laboratory, RIKEN GENESIS CO.,

29 LTD., Life Innovation Center, Kawasaki, Japan

3016 Genetic \& Pathology Department, SRL, Inc., Hachioji, Japan

3117 Development of Clinical Genomics, BML Inc., Kawagoe, Japan

3218 Molecular Genetic Analysis Department, Advanced Technology Center, LSI Medience Corporation,

33 Tokyo, Japan

3419 Second Department of Surgery, Hamamatsu University School of Medicine, Hamamatsu, Japan

3520 First Department of Surgery, Hamamatsu University School of Medicine, Hamamatsu, Japan

3621 Department of Diagnostic Pathology, Hamamatsu University School of Medicine, Hamamatsu,

37 Japan

38

$39 *$ Corresponding author

$40 \quad$ E-mail: mmaekawa@hama-med.ac.jp

41 


\section{Abstract}

43 To implement precision oncology, analytical validity as well as clinical validity and utility are

44 important. However, proficiency testing (PT) to assess validity has not yet been systematically

45 performed in Japan. To investigate the quality of next-generation sequencing (NGS) platforms and

46 cancer genome testing prevalent in laboratories, we performed pilot PT using patient samples. We

47 prepared 5 samples from patients with lung or colorectal cancer, extracted genomic DNA from the

48 cancer tissue and peripheral blood, and distributed these to 15 laboratories. Most participating

49 laboratories successfully identified the pathogenic variants, except for two closely located KRAS

50 variants, and 25-bp delins in the EGFR exon 19, not identified by the in vitro diagnostics testing.

51 Conversely, the EGFR L858R variant was successfully identified, and the allele frequency was similar

52 for all the laboratories. A high DNA integrity number led to excellent depth and reliable NGS results.

53 All NGS platforms and bioinformatics pipelines have advantages and disadvantages. We propose the

54 use of a PT program using patient samples to ascertain the quality status of cancer gene testing in

55 laboratories and to ensure that laboratories have sufficient information to develop advancements

56 in precision medicine for cancer. 


\section{Introduction}

59 The concept of personalized or precision medicine is expanding rapidly into practice and is based on

60 cancer biology, including somatic variants, in particular driver variants. It is entirely dependent on the

61 development of molecular methods, especially next-generation sequencing (NGS) technologies. NGS

62 and cancer gene panels can simultaneously analyze multiple gene variants through efficient DNA

63 sequencing and promote individualized treatment decision-making and precision medicine [1-3]. In

64 contrast, molecular genetic testing using NGS is very complex because it comprises nucleic acid

65 extraction, library preparation and sequencing chemistry, and bioinformatics pipelines. NGS

66 technologies and databases are continuing to evolve, but the interpretation of NGS data remains challenging; therefore, optimization of the NGS process is required to obtain the correct results that lead to adequate treatment. In Japan, cancer genome medicine has been promoted as a national strategy, and it is indispensable to ensure quality assurance for developing cancer genome testing. To implement precision oncology, analytical validity is as important as clinical validity and utility. Thus, to ensure suitable analytical validity, internal quality control and proficiency testing (PT) are essential. PT for cancer genome testing using NGS has been implemented using several types of testing specimens, primarily cell line-based samples and human genome DNA with spiked synthetic mutated DNA [1-10]. Yet, such PT has not been systematically performed in Japan. In this study, we have attempted to implement PT in patient samples to investigate the quality of NGS platforms and cancer genome testing usually used in laboratories. The purpose of this pilot PT is to ascertain the current quality status of cancer gene panel testing in Japan and disseminate the findings to stakeholders, including the participating laboratories, concerned academic societies, and policymakers. 


\section{Results}

The variants revealed using NGS analysis are described by each patient below, and the detailed information of the variants is shown in Supplementary Table 3.

\section{Patient no. 1 (rectal cancer, tumor density 50\%)}

Fig. 1 shows the proportions of adequate reports and variant allele frequency (VAF) distributions among laboratories. Ten laboratories (capture 4, amplicon 6) analyzed this sample. All the participating laboratories reported the $K R A S$ p.Gly13Asp (c.38G>A) missense variant, which led to a precise therapeutic strategy. KRAS is an actionable and druggable gene, and its pathological variants should be precisely identified. The variant was confirmed using the MEBGEN RASKET-B kit (PCR-rSSO). VAF values of the laboratories were similar (Fig. 1). The ddPCR indicated that the allele frequency was approximately $34 \%$, which was quite similar to that in the NGS analysis.

Two $A P C$ variants, p.Trp421Ter (c.1263G>A) and p.Asp1318ValfsTer5 (c.3948_3952 dupTGAAG) were reported by four and six laboratories, respectively. In about half of the participating laboratories, these $A P C$ variants were beyond the reportable range. The PDGFRA and CTNNB1 missense variants were reported by five laboratories.

The $B R C A 1$ missense germline variant, p.Met1008Val (c.3022A>G), was reported by four laboratories using the N/T pairs and one laboratory using $\mathrm{T}$ only. One laboratory detected the variant but did not report any clinical utility. The VAFs reported by the six laboratories were distributed around $50 \%$.

\section{Patient no. 2 (rectal cancer, tumor density $60 \%$ )}

Fourteen laboratories (capture 5, amplicon 9) analyzed this sample (Fig. 2). Ten laboratories reported the KRAS p.Lys117Asn (c.351A >C) variant, with two laboratories using the Oncomine Dx target test. The variant was revealed using RASKET-B kit. The allele frequencies of the variants were almost 
similar, and the ddPCR indicated that it was approximately $31 \%$, which was significantly lower than that of all participating laboratories.

The KRAS p.Leu120Met (c.358T>A) variant was reported by seven laboratories. One laboratory detected the variants but did not report any clinical utility, and four laboratories could not detect the variant by filtering out due to the bioinformatics pipeline. This variant was not included in the reportable range of the RASKET-B kit and Oncomine Dx target test.

These two variants in KRAS could be confirmed to be located on the same allele by visual inspection of the IGV file, which means that these variants do not cause two hits for both alleles. These variants were confirmed using the Sanger sequencing. The two variants were very close on the same allele and could not be easily identified using bioinformatics. Multiple variants at a close position might be programmed to be filtered out because of unreliable sequencing. Once the variant was removed, we could not confirm the IGV file visually, resulting in overlooking and false negatives. This result seems to be an issue of bioinformatics programs.

\section{Four other somatic variants were reported in several laboratories. In contrast, the ROSI nonsense} variant was reported to have an allele frequency of $21 \%$ from one laboratory but was not detected by the other laboratories. Sanger sequencing reports also confirmed negative data for this ROS1 variant (data not shown).

\section{Patient no. 3 (lung adenocarcinoma, tumor density 65\%)}

$$
\text { Eleven laboratories (capture 4, amplicon 7) analyzed this sample. Fig. } 3 \text { shows the tentative allele }
$$
frequencies of the detected variants. Five laboratories reported the deletion variants in EGFR exon 19; however, the deletion start site was different, resulting in additional amino-acid substitutions. In exon 19 of $E G F R$, many types of deletion variants have been discovered; there are several registries about these similar variants in COSMIC, as shown in Fig. 4. We performed Sanger sequencing and concluded that 
this patient had a complex variant of EGFR exon 19 (Supplementary Fig. 1). The correct answer was a combination of c.2252_2275del (COSMIC ID 23634) and c.2276T >A (I759N; ID 23633), or c.2252_2276delinsA (ID 96856) and was reported by two laboratories. Three laboratories reported the wrong answer, c.2253_2276del (ID 13556). The difference was only one nucleotide for the starting site of the 24-bp deletion. One laboratory reported the $1759 \mathrm{~N}$ (c.2276T>A) missense variant only. Other laboratories reported no variants in EGFR.

Two IVD reagents, the Therascreen EGFR mutation detection kit RGQ and Cobas EGFR Mutation Test v2, along with Oncomine Dx target test were designed to be able to identify some types of deletion variants in exon 19; however, the variants in patient 3 could not be precisely identified by the above tests and by most of the participating laboratories. The I759N missense variant is mostly pathogenic and should not be ignored. The 8-amino acid deletion and 1-amino acid substitution mostly damaged EGFR function and affected the selection of therapeutic agents; therefore, these variants should be successfully identified.

Somatic variants of TP53 and FGFR3 have not been interpreted for their clinical significance, and approximately half of the laboratories did not report these variants. The allele frequencies of these variants converged, and their CV\% was very small. One germline variant (STK11 p.Pro281Leu, c. $842 \mathrm{C}>\mathrm{T}$ ) was found in seven laboratories; however, the VAFs from four laboratories were slightly lower than the theoretical $50 \%$.

\section{Patient no. 4 (colon cancer, tumor density 25\%)}

Ten laboratories (capture 4, amplicon 6) analyzed this sample (Fig. 5). Eight laboratories reported the $B R A F$ V600E variant. Most laboratories detected and reported this variant; however, the allele frequency of this variant varied from $8 \%$ to $39 \%$ in laboratories using the amplicon-based procedure. Two laboratories using the Oncomine Dx target test did not report this variant, but variant reads were 
153

154

155

156

157

158

159

160

161

162

163

164

165

166

167

168

169

170

171

172

173

174

175

176

found in IGV files. This variant was confirmed using the IVD reagent, RASKET-B kit, and Sanger sequencing. Moreover, the ddPCR indicated that the allele frequency was approximately $20 \%$, which was close to the mean of the results from the reported laboratories.

The detection failure and variety in allele frequency might be explained by the following: the tumor density was the lowest (25\%), the FFPE of this tumor tissue was the oldest among the five testing samples, and the DNA integrity number (DIN) was the lowest (3.9). The dispersed VAF might cause this variant to be overlooked, especially around the detection limit; therefore, we should consider such cases for library preparation by PCR amplification and detection algorithm by bioinformatics in particular.

Many other variants have been identified and reported. This is probably a result of the dysfunction of the mismatch repair genes. Microsatellite instability (MSI) analysis indicated that all of the 5 mononucleotide repeat markers and one pentanucleotide repeat marker showed additional bands and were classified as the microsatellite instability-high (MSI-H) (Supplementary Fig. 2).

Immunohistochemistry analysis demonstrated positive staining of MSH2 and MSH6 and negative staining of MLH1 and PMS2 (Supplementary Fig. 3). These results are highly suggestive of the reduction of MLH1 expression.

It is reasonable that this sample had the somatic variant in $B R A F$ and that patient 4 was a woman in her sixties with adenocarcinoma in the ascending colon. Therefore, we suspected loss of function in $M L H 1$ by aberrant DNA methylation and revealed the aberrant hypermethylation at all ten $\mathrm{CpG}$ dinucleotide sites in the $\mathrm{CpG}$ island of $M L H 1$ (observed range of beta-value expressing methylation score: $0.52-$ $0.77 ; 0.016-0.11$ for normal accompanying mucosa) by DNA methylation analysis.

An important aspect of this case is the possibility of detecting this $B R A F$ V600E mutation. The $B R A F$ V600E variant has been well studied in many neoplasms, most commonly in melanoma, for which targeted therapies are available [11]. In colorectal cancer, $B R A F-\mathrm{V} 600 \mathrm{E}$ targeted therapies have been 
177 less successful, but $B R A F$-mutant metastatic colorectal cancer is associated with a worse prognosis.

178 Therefore, timely ascertainment of molecular subtypes is critically important for treatment planning and

179 earlier consideration of targeted therapies [12].

180

181 Patient no. 5 (lung adenocarcinoma, tumor density 60\%)

182 Fifteen laboratories (capture 6, amplicon 9) analyzed this sample. All 15 laboratories reported the

183 EGFR L858R variant, and the allele frequency of the variant was very similar (Fig. 6). Two IVD

184 reagents, the Therascreen EGFR mutation detection kit RGQ and Cobas EGFR Mutation Test v2, also

185 reported positive results. Moreover, the ddPCR indicated that the allele frequency was approximately

$18661 \%$, which was inside the distribution of the allele frequencies from the participating laboratories.

187 The EGFR A289V mutation was also reported by all participating laboratories with similar allele

188 frequencies. However, two IVD reagents did not report this variant, probably because the variant was

189 not within their reportable range.

190 Ten laboratories reported the TP53 F134L (p.Phe134Leu, c.402T >G) variant, and the allele

191 frequencies were similar. It was not included in the reportable range of the five laboratories. Twelve

192 laboratories reported the RET germline variant (p.Ser649Leu, c.1946C >T), but three laboratories using

193 the Oncomine Dx target test kit failed by reporting beyond the reportable range. This variant is

194 positioned in a conflicting interpretation of pathogenicity by ClinVar.

195

Relation between DNA quality and NGS result

We considered the effect of DNA quality on NGS results. Fig. 7a shows the correlation between the DNA quality and CV of allele frequencies. DNA prepared from FFPE with lower tumor density showed lower VAF and higher CV\% (patient 4, Fig. 5); low VAF tended to be inversely correlated with the $\mathrm{CV} \%$ of allele frequencies. Patient 3 presented a high $\mathrm{CV}$ of allele frequencies 

because of the 24-bp deletion and an adjacent single nucleotide variant, which seem to be unique. The ratio of depth among the laboratories against the DIN of each sample is shown in Fig. 7b. A high DIN

203 leads to excellent depth and possibly secure and reliable NGS results. The library preparation procedure

204 was not considered as an influencing factor. The DNA samples from patient 4 presented lower DIN

205 scores with significantly higher $\mathrm{CV} \%$ of allele frequencies $(p=0.032)$ and lower depth/AVERAGE

206 ratio $(p=0.00012)$ than the DNA samples from the other patients by the Student's $t$-test (Fig. 7a,b).

207 This implies that low DIN scores affect the quality of NGS results and might be descriptive of the

208 importance of preanalytical processes, especially DNA preparation.

209

210 


\section{Discussion}

212 In this study, we performed pilot PT study to share the current quality status of cancer gene panel

213 testing in Japan with any stakeholders. PT samples are usually derived from cell line-based samples [7]

214 or mixtures of the human genome and synthetic DNA-based samples [5,9]. Cell lines have the

215 advantage that they are prepared with known ratios to simulate different VAFs, however, they might be

216 expensive and time-consuming, or have sequence artifacts due to genetic manipulation and non-

217 physiological patterns $[4,6]$. Synthetic DNA fragments have the advantages that they can be designed to

218 harbor a broad range of sequence variants and can be used as spike-in standards, however, they may

219 have limited length resulting in artifacts being detected by NGS platforms $[4,6]$. The ideal PT samples

220 should be obtained from clinical specimens and should allow all phases of the testing process to be

221 evaluated. Yet, archival FFPE tissue specimens are not easily available for large-scale PT studies

222 because of the limited number of tumor and normal tissue samples available from a given patient [5].

223 For initial PT in Japan, we selected five pairs of patients with cancer, human genomic DNA prepared

224 from the tumor (FFPE), and normal cells (peripheral blood). Such matched pairs of cancer and normal

225 DNA are very useful for comparisons with the NGS platform and bioinformatics pipeline. Our concept

226 focuses on the ACCE model for evaluating genetic tests using CDC [13]. The NGS technology consists

227 of preanalytical, analytical, and post-analytical processes, similar to any other specimen examination.

228 Therefore, we distributed purified DNA for PT samples to compare only NGS analysis findings and to

229 avoid differences among laboratories with respect to DNA purification quality and the portion of FFPE.

230 We prepared both tumor and normal DNA samples in this PT study, and a few laboratories analyzed

231 reported DNA germline variants in patients (nos. 1, 3, and 5). The VAFs of the variants were distributed

232 amongst around $50 \%$ of the patients (nos. 1 and 5); however, the VAFs from four laboratories were

233 nearly $30 \%$. The reason remains unclear; however, three of the four laboratories used amplicon-based

234 library preparation, resulting in PCR bias due to reasons such as nucleotide alignment near the primer 
annealing and GC contents. One laboratory that reported about 30\% of VAFs were estimated using a capture-based method with molecular barcodes. The read depth of the variant was not lower than that of the other variants, and the reason for the lower VAF was not known.

The accuracy of NGS analysis depends on the quality, integrity, and amount of nucleic acids used as well as the NGS platform and the use of amplicon-based or capture-based methods. In the present PT trial, we used the same prepared DNA solution, and we focused on the performance of the NGS workflow used in each laboratory. DNA prepared from FFPE with lower tumor density showed lower VAF and higher CV\%, shown in Fig. 7 a and b. Thus, DNA quality affects the NGS results, and the preanalytical processes, especially cancer tissue control and DNA preparation, are strictly important.

\section{Generally, the major variants tended to be adequately reported; however, certain variants could not be} precisely detected in some laboratories. Most laboratories specified the variants with clinical utility but in some samples when multiple variants were closely located or had complex deletion variants they were difficultly revealed. However, Onozawa et al. [14] identified the EGFR L858R-K860I doublet mutation using an NGS-based PT, which could not be detected by an initial PCR-based companion test, thus resulting in successful treatment. These detection failures may be primarily caused by the bioinformatics pipeline. Reportable ranges were different, and variants reported because of their clinical significance were also different in each laboratory. Conflicting interpretations of pathogenicity and variants of uncertain significance should be reduced with the improvement of variant databases.

Moreover, an easily understandable, useful, and precise report format by molecular pathologists, laboratory physicians, medical scientists, and bioinformaticians is necessary, and standardization of the interpretation and reporting in cancer genomic medicine should be considered [15].

We recognize the real abilities and weaknesses of our NGS analysis as this was an extremely valuable educational opportunity of the PT study. One strength of this pilot PT is knowledge on the current quality of any NGS platforms and cancer genome testing usually used in laboratories. However, there 
259 are some limitations associated with our study: the correct answers of each specimen were not perfectly

260 decided; the variants detected in these randomly selected patients may not necessarily be represented in

261 the real world of oncology; and the number of the participating laboratories is small.

262 We recognize the strengths and weaknesses of the NGS platform, including wet and dry processes in

263 the laboratories. If important druggable variants are not detected, they cannot be applied in genotype-

264 matched treatments. In other words, analytical validity is indispensable, and this issue should be

265 addressed in future analysis systems. Therefore, we expect that we would have a unique PT for

266 precision cancer genomic medicine. Participation in PT is an opportunity to recognize the pros and cons

267 of the NGS analysis system used in the laboratory. These findings serve as reference data for assessing

268 the improvement in precision oncology in the future.

269

270 Conclusion

271 There are many NGS platforms and bioinformatics pipelines for cancer genome analysis. Thus, the

272 implementation of PT is indispensable to recognize the present quality status of our own laboratory and

273 any other laboratories and to highlight the importance of quality assurance in cancer genome testing. We

274 propose the use of PT using patient samples to understand the real-world applications in oncology and

275 to educate participant laboratories the advantages and disadvantages of the actual NGS platform.

276

277 


\section{Materials and Methods}

\section{Samples for $P T$}

Patient samples, obtained from the Department of Diagnostic Pathology at Hamamatsu University Hospital, comprised formalin-fixed paraffin-embedded (FFPE) tissues from five patients with lung or colorectal cancer; matched normal samples were obtained from peripheral blood. These patients were selected based on their relatively large tumor masses and on providing informed consent. The clinical and pathological features of the patients are presented in Supplementary Table 1. The histological findings were also supplied to the participating laboratories.

The genomic DNA (gDNA) was prepared using the QIAamp DNA FFPE Tissue Kit and Deparaffinization Solution (QIAGEN) from FFPE and the EZ1 DNA Blood Kit (QIAGEN, Hilden, Germany) from the whole-blood. The DNA quantity and quality were measured using a 2200 TapeStation (Agilent Technologies, Santa Clara, CA) system using the TapeStation Analysis software (Agilent), which automatically determines and displays the DNA Integrity Number (DIN). The properties of the DNA samples are presented in Table 1.

This study was approved by the Institutional Review Board of the Hamamatsu University School of Medicine (19-186). Five pairs of FFPE and peripheral blood samples were prepared with the patients' written informed consent. All methods were carried out in accordance with the Declaration of Helsinki. We tentatively decided that two samples (nos. 2 from colorectal cancer and 5 from lung cancer) were essential for all laboratories, and the remaining three samples (patient nos. 1, 3, and 4) were optional. Each participating laboratory selected either the two essential samples, all five samples, or another set of samples based on their NGS platform or financial reasons. PT samples were sent to the participating laboratories on March 24, 2020, with information about the DNA quality and quantity of the samples and the patients' pathological and clinical findings. 


\section{PT participants}

Fifteen clinical laboratories (12 hospital clinical laboratories and 3 registered clinical laboratories) participated in this study. The sequencing assay examined in this study was independently validated and implemented in each laboratory. The assay platform, bioinformatics pipeline, and other procedures differed among laboratories. Five laboratories used the hybrid capture-based method, and 10 laboratories used the amplicon-based method for NGS library preparation. Seven laboratories analyzed paired samples of tumor $(\mathrm{T})$ tissues and normal blood cells $(\mathrm{N})$, and eight used tumor tissues only. Supplementary Table 2 provides a brief explanation of the laboratories and the analytic procedure. The analysis platform, gene panel (targeted genes, all coding exons or hotspot only), bioinformatics pipeline, and reportable range were different among the laboratories.

We collected the analysis reports from all participating laboratories first at the end of May 2020 and then asked whether the consensus mutations could be detected by NGS analysis and the reason in case they were not reported, including no clinical utility.

\section{Additional analysis method for the PT samples}

The PT specimens were also analyzed using in vitro diagnostics (IVD) reagents in a commercial laboratory accredited by ISO 15189 and CLIA. For lung cancer, the EGFR variants were analyzed using the Therascreen EGFR mutation detection kit RGQ based on Scorpion-ARMS (QIAGEN) and Cobas EGFR Mutation Test v2 (Roche Diagnostics, Basel, Switzerland) based on a real-time PCR test. For colorectal cancer, the $R A S$ and $B R A F$ variants were examined using the MEBGEN RASKET-B kit based on PCR-reverse sequence-specific oligonucleotide (PCR-rSSO) (MBL, Nagoya, Japan) and a Cobas BRAF V600 detection kit based on real-time PCR (Roche Diagnostics).

The allele frequencies of the major variants were quantified using the droplet digital PCR (ddPCR). The ddPCR was performed using a QX200 AutoDG Droplet Digital PCR system (Bio-Rad Laboratories 
327 ddPCR experiments were performed in triplicate.

328 Sanger sequencing was performed using the BigDye Terminator v3.1 cycle sequencing Kit (ABI, Foster 329 City, CA) and the ABI 3500xL Genetic Analyzer (ABI). Microsatellite instability (MSI) was detected by 330 a Promega MSI Analysis System with five mononucleotide repeat markers (BAT-26, BAT-25, NR-21,

331 NR-24 and MONO-27) according to the manufacturer's protocol (Promega, Madison, WI). Samples

332 with $\geq 2$ altered markers out of 5 were classified as MSI-H. Two pentanucleotide repeat markers (Penta-

333 C and Penta-D) were used to confirm that the test sample and the paired normal samples were from the

334 same individual.

335 Immunohistochemistry staining for the expression of 4 mismatch repair proteins (MLH1, MSH2,

336 PMS2 and MSH6) was performed as described previously [16]. DNA methylation analysis was

337 performed using the Infinium MethylationEPIC BeadChip Kit (Illumina, San Diego, CA) according to

338 the manufacturer's protocol.

339

$340 \quad$ Statistical analysis

341 Statistical analyses were performed using Microsoft Excel 2017 for Windows, and $P<0.05$ was

342 considered statistically significant. 
345 1. Pfeifer, J. D. Clinical next generation sequencing in cancer. Cancer Genet. 206, 409-412 (2013).

346 2. Surrey, L. F., et al. Next-generation sequencing (NGS) methods show superior or equivalent 347 performance to non-NGS methods on BRAF, EGFR, and KRAS proficiency testing samples. Arch. $348 \quad$ Pathol. Lab. Med. 143, 980-984 (2019).

349 3. Singh, R. R. Next-generation sequencing in high-sensitive detection of mutations in tumors: $350 \quad$ challenges, advances, and applications. J. Mol. Diagn. 22, 994-1007 (2020).

351 4. Kalman, L. V., et al. Current landscape and new paradigms of proficiency testing and external quality assessment for molecular genetics. Arch. Pathol. Lab. Med. 137, 983-988 (2013).

5. Zhang, R., et al. The reliable assurance of detecting somatic mutations in cancer- related genes by next-generation sequencing: the results of external quality assessment in China. Oncotarget 7, $58500-58515$ (2016).

6. Duncavage, E. J., et al. A model study of in silico proficiency testing for clinical next-generation sequencing. Arch. Pathol. Lab. Med. 140, 1085-1091 (2016).

7. Richman, S. D., et al. Results of the UK NEQAS for Molecular Genetics reference sample analysis. J. Clin. Pathol. 71, 989-994 (2018).

8. Tack, V., et al. Describing the reportable range is important for reliable treatment decisions: A multiple laboratory study for molecular tumor profiling using next-generation sequencing. J. Mol. Diagn. 20, 743-753 (2018).

9. Merker, J. D., et al. Proficiency testing of standardized samples shows very high interlaboratory agreement for clinical next-generation sequencing-based oncology assays. Arch. Pathol. Lab. Med. 143, 463-471 (2019). in Ontario: A pilot study. Curr. Oncol. 26, e717-e732 (2019). 
11. Ritterhouse, L. L. \& Barletta, J. A. BRAF V600E mutation-specific antibody: a review. Semin. Diagn. Pathol. 32, 400-408 (2015).

12. Chu, J. E., et al. Population-based screening for $B R A F^{\mathrm{v} 600 \mathrm{E}}$ in Metastatic Colorectal Cancer Reveals Increased Prevalence and Poor Prognosis. Clin. Cancer Res. 26, 4599-4605 (2020).

13. Haddow, J. \& Acce, P. G. A model process for evaluating data on emerging genetic tests in (eds Khoury, M., Little, J. \& Burke, W.). Human Genome Epidemiology: A Scientific Foundation for Using Genetic Information to Improve Health and Prevent Disease (Oxford: Oxford University Press, 2004) 217-233

14. Onozawa, H., et al. Lung adenocarcinoma in a patient with a cis EGFR L858R-K860I doublet mutation identified using NGS-based profiling test: negative diagnosis on initial companion test and successful treatment with Osimertinib. Thorac. Cancer 11, 3599-3604 (2020).

15. Li, M. M., et al. Standards and guidelines for the interpretation and reporting of sequence variants in cancer: A joint consensus recommendation of the Association for Molecular Pathology, American Society of Clinical Oncology, and College of American Pathologists. J. Mol. Diagn. 19, 4-23 (2017). endoscopic submucosal dissection. Oncol. Lett. 20, 2435-2441 (2020). 


\section{Acknowledgments}

387 The authors appreciate the assistance provided by all participating staff members and laboratories.

This work was supported by the Japan Society for the Promotion of Science (KAKENHI grant no.

20K07823, and Pfizer Global Medical Grants (ID\# 54439477). MM has received grants provided to the Department of Laboratory Medicine, Hamamatsu University School of Medicine from Sysmex Co. KN has received grants provided to the Kindai University Faculty of Medicine from Pfizer (ID\#54954951). We would like to thank Editage (www.editage.com) for English language editing.

\section{Author contributions}

MM, MI, KN, KS, KM and KN contributed to the study design and conducted this project. MI, MM, KK, KF and SB secured IRB approval and collected samples. MM, TT and MI prepared the samples for distribution. MM, TT, KN, KS, KM, TI, KN, MI, HN, KS, YY, KCH, YH, YY, KF, SO, KS, MY, YK, HS, YT, MF, TY, KM and MI analyzed the samples. MM and MI wrote the manuscript and all authors reviewed and approved the final version of the manuscript.

\section{Competing interests}

MM has received grants provided to the Department of Laboratory Medicine, Hamamatsu University School of Medicine from Pfizer (ID\# 54439477) and Sysmex Co. KN has received grants provided to the Kindai University Faculty of Medicine from Pfizer (ID\#54954951). YK, HS, YT, MF, TY and KM are employees at their corresponding affiliation. The other authors have no relevant financial interest in the products or companies described in this article. 
410 Medicine (19-186) according to the ethical guidelines of the Japanese government. Written informed 411 consent was obtained from the individuals for obtaining samples of FFPE and peripheral blood. All 412 methods were carried out in accordance with the Declaration of Helsinki.

413 


\section{$414 \quad$ Figure Legends}

\section{Figure 1. Report summary of patient 1}

417 Major variants reported by the laboratories and variant allele frequency (VAF) concordance with the 418 coefficient of variation $(\mathrm{CV})$ are shown.

419 a) Variants reported by the participating laboratories are presented, and information about the variants is 420 presented in the following panels b), c) and d). The variants highlighted in bold should be detected 421 because of their relationship with therapeutics. (G) represents the germline variant. b) Report status for the variants indicated in a) are presented. "1st time" indicates reporting at the first time without information, "2nd time" indicates reporting after the question regarding the existence of the variant, and "not detected" indicates no reporting after the question.

c) VAF of the reported variants is presented. Open diamonds and closed circles indicate capture-based and amplicon-based methods, respectively. The vertical bar indicates the ddPCR results, assayed in triplicate.

d) CVs (\%) of the VAF in the laboratory reports are shown.

\section{Figure 2. Report summary of patient 2}

431 Major variants reported by the laboratories and VAF concordance with the CV for patient 2 are shown.

432 a) Variants reported by the participating laboratories are presented, and information about the variants is presented in the following panels b), c) and d).

b) Report status for the variants indicated in a) are presented. "1st time" indicates reporting at the first time without information, "2nd time" indicates reporting after the question regarding the existence of

436 the variant, and "not detected" indicates no reporting after the question.

437 c) VAF of the reported variants is presented. Open diamonds and closed circles indicate capture-based 

triplicate.

d) CVs (\%) of the VAF in the laboratory reports are shown.

Figure 3. Report summary of the patient 3.

443 Major variants reported by the laboratories and VAF concordance with the CV for the patient 3 are 444 shown.

445 a) Variants reported by the participating laboratories are presented, and information about the variants is 446 presented in the following panels b), c) and d). The variants highlighted in bold should be detected because of their relationship with therapeutics. (G) represents the germline variant. b) Report status for the variants indicated in a) are presented. "1st time" indicates reporting at the first time without information, "2nd time" indicates reporting after the question regarding the existence of the variant, and "not detected" indicates no reporting after the question.

c) VAF of the reported variants is presented. Open diamonds and closed circles indicate capture-based and amplicon-based methods, respectively.

d) CVs (\%) of the VAF in the laboratory reports are shown.

\section{Figure 4. Deletion variant in EGFR exon 19 in patient 3}

456 The variant results obtained from NGS and Sanger sequencing (Supplementary Fig. 1) are summarized. Five types of variants were reported. Deletion variants identified by laboratories were slightly different. However, the correct answer is COSMIC ID 96856 or ID $23634+232633$, which causes deletion of 8 amino acids and a $\mathrm{T}$ to $\mathrm{N}$ amino acid substitution, resulting in a possibly pathogenic variant. 
462 Major variants reported by the laboratories and VAF concordance with the CV for the patient 4 are

463 shown.

464 a) Variants reported by the participating laboratories are presented, and information about the variants is

465 presented in the following panels b), c) and d). The variants highlighted in bold should be detected

466 because of their relationship with therapeutics.

467 b) Report status for the variants indicated in a) are presented. " 1 st time" indicates reporting at the first 468 time without information, "2nd time" indicates reporting after the question regarding the existence of 469 the variant, and "not detected" indicates no reporting after the question.

470 c) VAF of the reported variants is presented. Open diamonds and closed circles indicate capture-based 471 and amplicon-based methods, respectively. The vertical bar indicates the ddPCR results, assayed in 472 triplicate.

473 d) $\mathrm{CVs}(\%)$ of the VAF in the laboratory reports are shown.

Figure 6. Report summary of patient 5.

476 Major variants reported by the laboratories and VAF concordance with the CV for the patient 5 are 477 shown.

478 a) Variants reported by the participating laboratories are presented, and information about the variants is 479 presented in the following panels b), c) and d). The variants highlighted in bold should be detected because of their relationship with therapeutics. $(G)$ represents the germline variant.

b) Report status for the variants indicated in a) are presented. "1st time" indicates reporting at the first time without information, "2nd time" indicates reporting after the question regarding the existence of the variant, and "not detected" indicates no reporting after the question.

c) VAF of the reported variants is presented. Open diamonds and closed circles indicate capture-based and amplicon-based methods, respectively. The vertical bar indicates the ddPCR results, assayed in 
486 triplicate.

487 d) CVs (\%) of the VAF in the laboratory reports are shown.

488

489 Figure 7. Correlation between DNA quality and NGS results.

490 a) Correlation between DNA quality and $\mathrm{CV}$ of allele frequencies for each mutation. Arrows and

491 numbers indicate the number of patient samples. Each circle indicated the CV of allele frequencies of 492 the detected variants.

493 b) Correlation between DNA quality and depth of sequencing coverage for each laboratory. Arrows and 494 numbers indicate the patient samples. Open and closed circles indicate the capture-based and amplicon495 based methods, respectively.

496

497 
Table 1. Properties of the patient samples

\begin{tabular}{|c|c|c|c|c|c|c|c|c|}
\hline & $\begin{array}{l}\text { Patient } \\
\text { ID }\end{array}$ & Origin & $\begin{array}{l}\text { Tumor } \\
\text { density } \\
(* 1)\end{array}$ & $\begin{array}{l}\text { Cancer } \\
\text { location }\end{array}$ & $\begin{array}{c}\text { DNA } \\
\text { quantity } \\
(* 2) \\
(\mathrm{ng} / \mu \mathrm{L})\end{array}$ & $\begin{array}{l}\text { Distributed } \\
\text { sample } \\
\text { volume } \\
(\mu \mathrm{L})\end{array}$ & $\begin{array}{c}\text { Optical } \\
\text { density ratio } \\
(260 \mathrm{~nm} / 280 \\
\mathrm{nm})(* 3)\end{array}$ & $\begin{array}{l}\text { DIN } \\
(* 4)\end{array}$ \\
\hline \multirow{4}{*}{$\begin{array}{l}\text { Essential } \\
\text { examination }\end{array}$} & $2 \mathrm{~T}$ & FFPE & $60 \%$ & Rectum & 241.0 & 10 & 2.02 & 5.5 \\
\hline & $2 \mathrm{~N}$ & Blood & & & 85.4 & 10 & 1.87 & 9.8 \\
\hline & $5 \mathrm{~T}$ & FFPE & $60 \%$ & Lung & 209.0 & 10 & 1.98 & 5.5 \\
\hline & $5 \mathrm{~N}$ & Blood & & & 78.8 & 10 & 1.79 & 9.5 \\
\hline \multirow{6}{*}{$\begin{array}{l}\text { Optional } \\
\text { examination }\end{array}$} & $1 \mathrm{~T}$ & FFPE & $50 \%$ & Rectum & 380.0 & 10 & 2.01 & 5.4 \\
\hline & $1 \mathrm{~N}$ & Blood & & & 79.0 & 10 & 1.80 & 9.7 \\
\hline & $3 \mathrm{~T}$ & FFPE & $65 \%$ & Lung & 172.0 & 10 & 1.97 & 5.8 \\
\hline & $3 \mathrm{~N}$ & Blood & & & 56.7 & 10 & 1.82 & 9.3 \\
\hline & $4 \mathrm{~T}$ & FFPE & $25 \%$ & Colon & 168.0 & 10 & 1.90 & 3.9 \\
\hline & $4 \mathrm{~N}$ & Blood & & & 108.0 & 10 & 1.83 & 9.1 \\
\hline
\end{tabular}




\section{Figures}

(a)

Patient 1

Rectum cancer

Tumor density $=50 \%$

\section{KRAS p.Gly13Asp c. $38 \mathrm{G}>\mathrm{A}$}

APC p.Trp421Ter c. $1263 \mathrm{G}>\mathrm{A}$

APC p.Asp1318ValfsTer5 c.3948_3952dup

PDGFRA p.Arg804GIn c. $2411 \mathrm{G}>\mathrm{A}$

CTNNB1 p.Arg565Cys c. $1693 \mathrm{C}>\mathrm{T}$

BRCA1 (G) p.Met1008Val c. $3022 A>G$ (b)

\begin{tabular}{|c|c|c|c|}
\hline \multicolumn{4}{|c|}{ Laboratory number } \\
\hline \multicolumn{3}{|c|}{ Report } & \multirow{2}{*}{$\begin{array}{l}\text { out of } \\
\text { reportable } \\
\text { range }\end{array}$} \\
\hline $\begin{array}{l}\text { 1st } \\
\text { time }\end{array}$ & $\begin{array}{l}\text { 2nd } \\
\text { time }\end{array}$ & $\begin{array}{c}\text { not } \\
\text { detected }\end{array}$ & \\
\hline 10 & 0 & 0 & 0 \\
\hline 4 & 0 & 1 & 5 \\
\hline 6 & 0 & 0 & 4 \\
\hline 5 & 1 & 0 & 4 \\
\hline 5 & 0 & 0 & 5 \\
\hline 5 & 1 & 0 & 4 \\
\hline
\end{tabular}

(c)

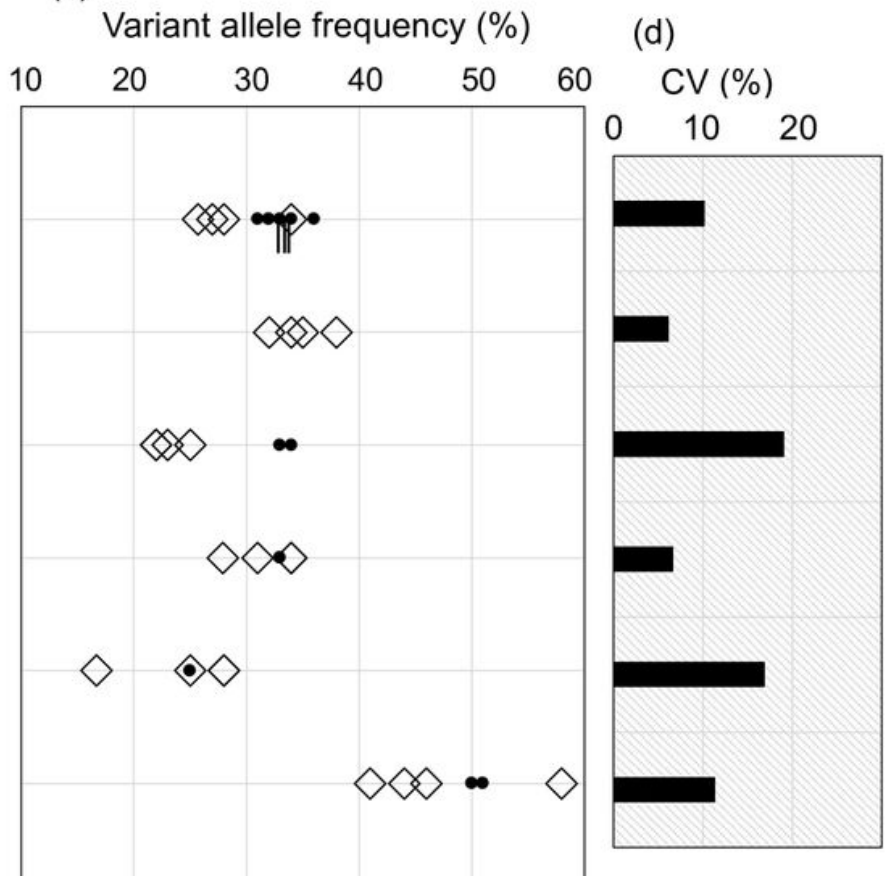

Fig. 1

\section{Figure 1}

Report summary of patient 1 Major variants reported by the laboratories and variant allele frequency (VAF) concordance with the coefficient of variation (CV) are shown. a) Variants reported by the participating laboratories are presented, and information about the variants is presented in the following panels b), c) and d). The variants highlighted in bold should be detected because of their relationship with therapeutics. (G) represents the germline variant. b) Report status for the variants indicated in a) are presented. "1st time" indicates reporting at the first time without information, "2nd time" indicates reporting after the question regarding the existence of the variant, and "not detected" indicates no reporting after the question. c) VAF of the reported variants is presented. Open diamonds and closed circles indicate capture-based and amplicon-based methods, respectively. The vertical bar indicates the ddPCR results, assayed in triplicate. d) CVs (\%) of the VAF in the laboratory reports are shown. 
(a)

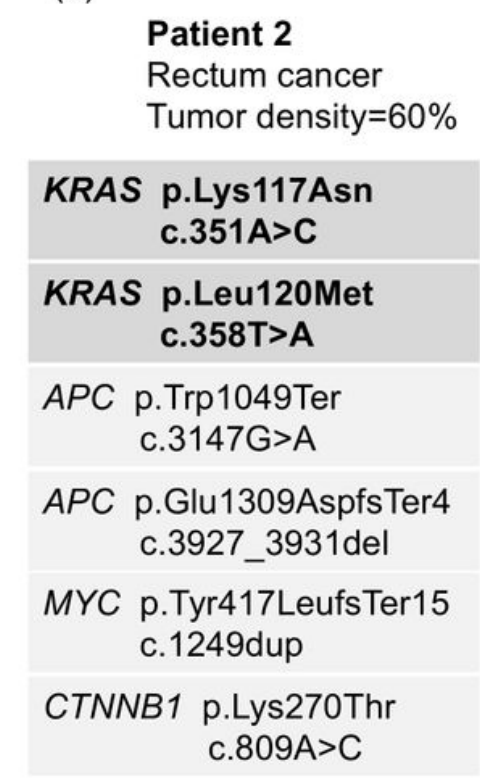

(b)

\begin{tabular}{|c|c|c|c|}
\hline \multicolumn{4}{|c|}{ Laboratory number } \\
\hline \multicolumn{3}{|c|}{ Report } & \multirow{2}{*}{$\begin{array}{c}\text { out of } \\
\text { reportable } \\
\text { range }\end{array}$} \\
\hline $\begin{array}{c}1 \mathrm{st} \\
\text { time }\end{array}$ & $\begin{array}{l}\text { 2nd } \\
\text { time }\end{array}$ & $\begin{array}{c}\text { not } \\
\text { detected }\end{array}$ & \\
\hline 10 & 3 & 1 & 0 \\
\hline 7 & 5 & 0 & 2 \\
\hline 8 & 0 & 0 & 6 \\
\hline 9 & 0 & 0 & 5 \\
\hline 5 & 2 & 1 & 6 \\
\hline 7 & 0 & 1 & 6 \\
\hline
\end{tabular}

(c)

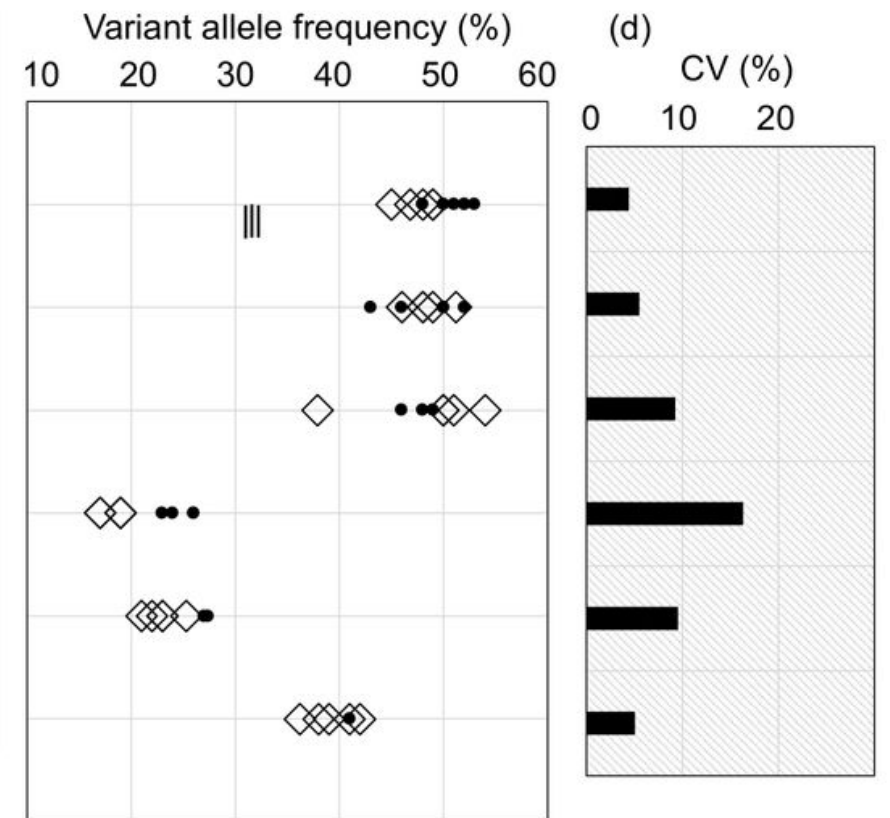

Fig. 2

\section{Figure 2}

Report summary of patient 2 Major variants reported by the laboratories and VAF concordance with the CV for patient 2 are shown. a) Variants reported by the participating laboratories are presented, and information about the variants is presented in the following panels b), c) and d). b) Report status for the variants indicated in a) are presented. "1st time" indicates reporting at the first time without information, "2nd time" indicates reporting after the question regarding the existence of the variant, and "not detected" indicates no reporting after the question. c) VAF of the reported variants is presented. Open diamonds and closed circles indicate capture-based and amplicon-based methods, respectively. The vertical bar indicates the ddPCR results, assayed in triplicate. d) CVs (\%) of the VAF in the laboratory reports are shown. 


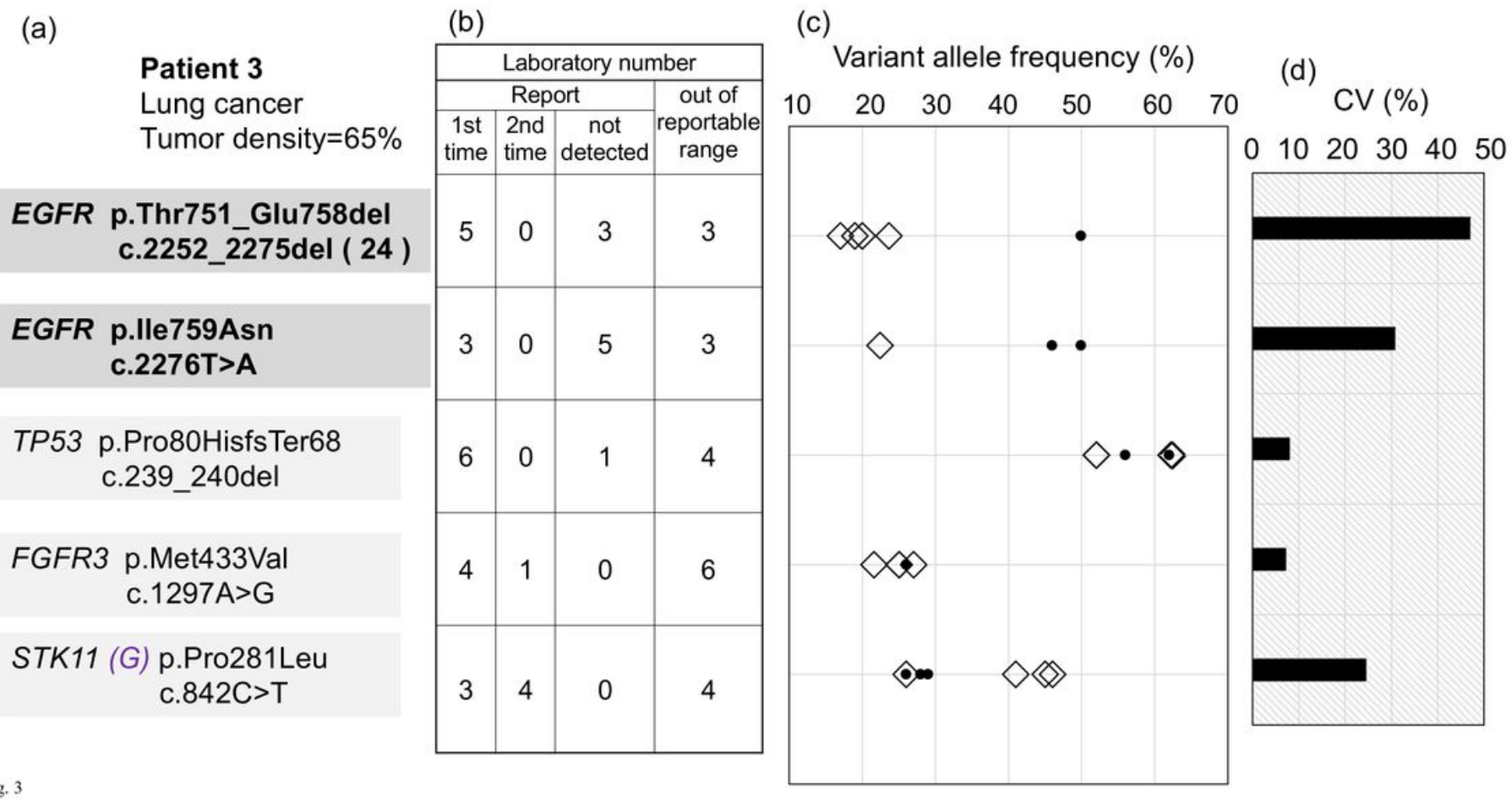

\section{Figure 3}

Report summary of the patient 3. Major variants reported by the laboratories and VAF concordance with the $\mathrm{CV}$ for the patient 3 are shown. a) Variants reported by the participating laboratories are presented, and information about the variants is presented in the following panels b), c) and d). The variants highlighted in bold should be detected because of their relationship with therapeutics. (G) represents the germline variant. b) Report status for the variants indicated in a) are presented. "1st time" indicates reporting at the first time without information, "2nd time" indicates reporting after the question regarding the existence of the variant, and "not detected" indicates no reporting after the question. c) VAF of the reported variants is presented. Open diamonds and closed circles indicate capture-based and ampliconbased methods, respectively. d) CVs (\%) of the VAF in the laboratory reports are shown. 


\begin{tabular}{|c|c|c|c|c|c|c|c|c|c|c|c|c|c|c|c|c|}
\hline \multirow{3}{*}{$\begin{array}{l}\text { No. of } \\
\text { reports }\end{array}$} & \multirow{3}{*}{$\begin{array}{c}\text { cosmic } \\
\text { ID }\end{array}$} & \multirow{3}{*}{ HGVSc } & \multirow{3}{*}{ HGVSp } & \multicolumn{11}{|c|}{ Amino acid coding number: hg19 } & \multirow{3}{*}{$\begin{array}{c}\text { COSMIC } \\
\text { Significance }\end{array}$} & \multirow{3}{*}{$\begin{array}{c}\text { ClinVar } \\
\text { Significance }\end{array}$} \\
\hline & & & & 750 & 751 & 752 & 753 & 754 & 755 & 756 & 757 & 758 & 759 & 760 & & \\
\hline & & & & A & $\mathrm{T}$ & s & $\mathbf{P}$ & $\mathrm{K}$ & A & $\mathbf{N}$ & $\mathbf{K}$ & $\mathbf{E}$ & 1 & $\mathrm{~L}$ & & \\
\hline 3 & 13556 & c.2253_2276del & p.Ser752_Ile759del & A & $\mathrm{T}$ & - & - & - & - & - & - & - & - & L & $\mathrm{n} / \mathrm{a}$ & drug response \\
\hline 0 & 6256 & c.2254_2277del & p.Ser752_Ile759del & A & T & - & - & - & 一 & - & - & - & - & L & $\mathrm{n} / \mathrm{a}$ & drug response \\
\hline 1 & 96856 & c.2252_2276delinsA & p.Thr751_Ile759delinsN & A & - & - & - & - & - & 一 & - & - & $\mathrm{N}$ & L & $\mathrm{n} / \mathrm{a}$ & \\
\hline \multirow{2}{*}{1} & 23634 & c. $2252 \_2275 \mathrm{del}$ & p.Thr751_Glu758del & A & - & - & - & - & - & - & - & - & \multirow[t]{2}{*}{$\mathrm{N}$} & \multirow[t]{2}{*}{$\mathrm{L}$} & $\mathrm{n} / \mathrm{a}$ & \\
\hline & 23633 & c. $2276 \mathrm{~T}>\mathrm{A}$ & p.lle759Asn & & & & & & & & & & & & Pathogenic & \\
\hline 1 & 23633 & c. $2276 \mathrm{~T}>\mathrm{A}$ & p.lle759Asn & A & $\mathrm{T}$ & S & $P$ & $\mathrm{~K}$ & A & $\mathrm{N}$ & $\mathrm{K}$ & $E$ & $\mathrm{~N}$ & L & Pathogenic & \\
\hline
\end{tabular}

Fig. 4

\section{Figure 4}

Deletion variant in EGFR exon 19 in patient 3 The variant results obtained from NGS and Sanger sequencing (Supplementary Fig. 1) are summarized. Five types of variants were reported. Deletion variants identified by laboratories were slightly different. However, the correct answer is COSMIC ID 96856 or ID $23634+232633$, which causes deletion of 8 amino acids and a T to $\mathrm{N}$ amino acid substitution, resulting in a possibly pathogenic variant. 
(a)

Patient 4

Ascending colon cancer

Tumor density $=25 \%$

BRAF p.Val600Glu

APC p.Thr1556Asn fsTer3

APC p.Asp849lle fsTer12

FBXW7 p.Arg465Cys

PALB2 p.Phe612Ser fsTer4

ALK p.Ala1300Val

PDGFRA p.Ala401Val

TSC1 p.Glu860Lys

BRCA2 p.Asp364Asn

JAK1 p.Lys860Asn fsTer16

CREBBP p.Asn722Asp (b)

\begin{tabular}{|c|c|c|c|}
\hline \multicolumn{4}{|c|}{ Laboratory number } \\
\hline \multicolumn{3}{|c|}{ Report } & $\begin{array}{c}\text { out of } \\
\text { 1st } \\
\text { time }\end{array}$ \\
\begin{tabular}{c|c|c|} 
time \\
time
\end{tabular} & $\begin{array}{c}\text { not } \\
\text { detected }\end{array}$ & $\begin{array}{c}\text { range } \\
\text { range }\end{array}$ \\
\hline 8 & 1 & 1 & 0 \\
\hline 5 & 1 & 0 & 4 \\
\hline 4 & 0 & 1 & 5 \\
\hline 7 & 0 & 0 & 3 \\
\hline 5 & 0 & 1 & 4 \\
\hline 5 & 1 & 0 & 4 \\
\hline 5 & 0 & 1 & 4 \\
\hline 5 & 1 & 0 & 4 \\
\hline 5 & 1 & 0 & 4 \\
\hline 4 & 0 & 1 & 5 \\
\hline 5 & 1 & 0 & 4 \\
\hline
\end{tabular}

(c)

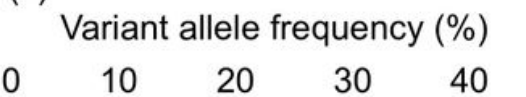

(d) CV (\%)

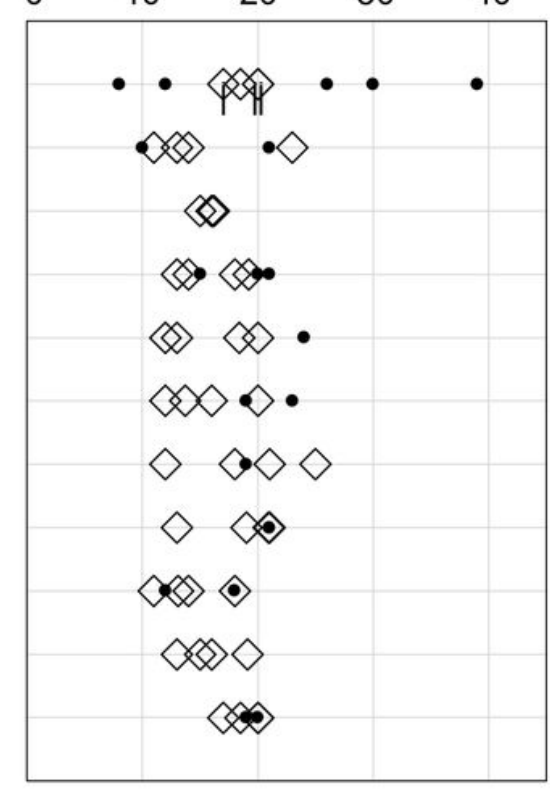

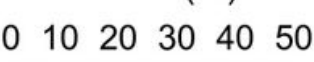

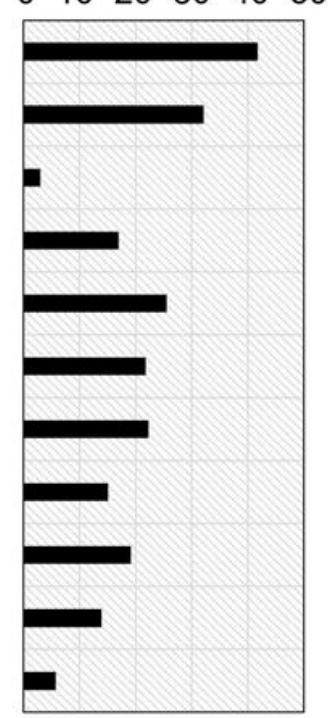

Fig. 5

\section{Figure 5}

Report summary of patient 4. Major variants reported by the laboratories and VAF concordance with the CV for the patient 4 are shown. a) Variants reported by the participating laboratories are presented, and information about the variants is presented in the following panels b), c) and d). The variants highlighted in bold should be detected because of their relationship with therapeutics. b) Report status for the variants indicated in a) are presented. "1st time" indicates reporting at the first time without information, "2nd time" indicates reporting after the question regarding the existence of the variant, and "not detected" indicates no reporting after the question. c) VAF of the reported variants is presented. Open diamonds and closed circles indicate capture-based and amplicon-based methods, respectively. The vertical bar indicates the ddPCR results, assayed in triplicate. d) CVs (\%) of the VAF in the laboratory reports are shown. 
(a)

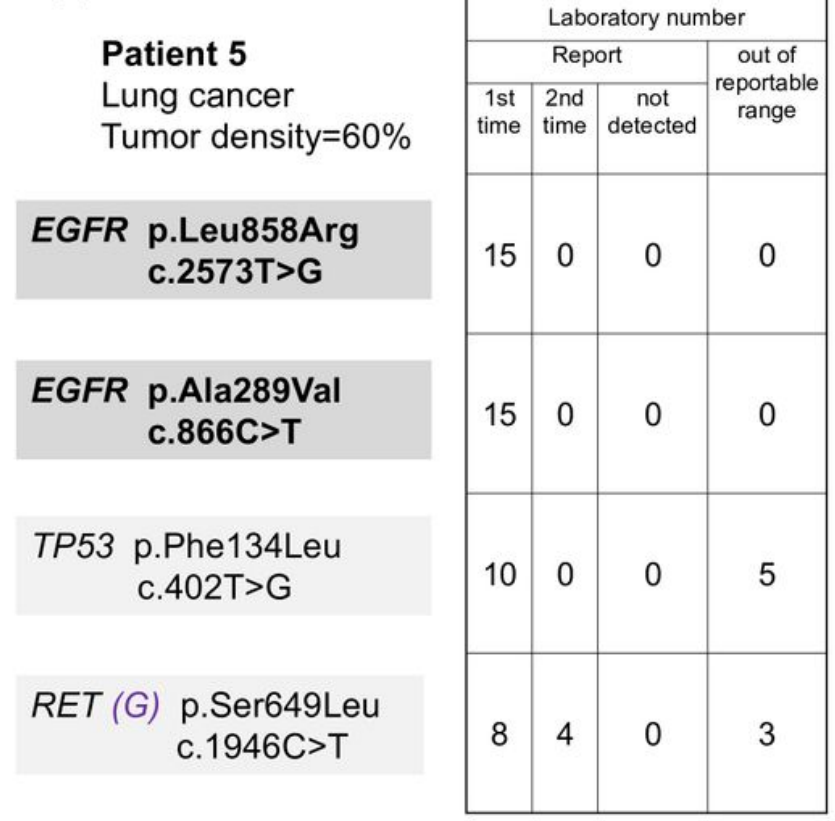

Fig. 6 (b)

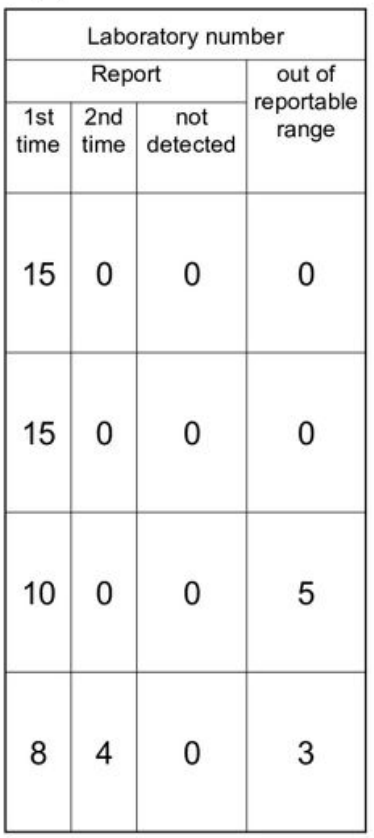

(c)

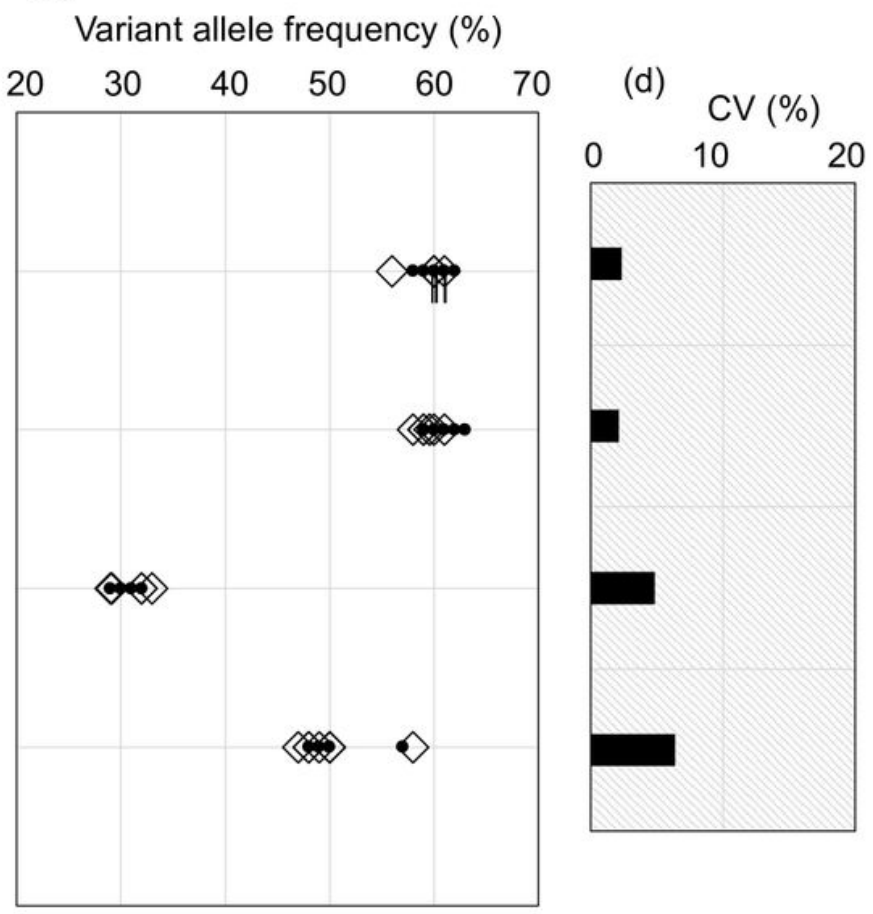

\section{Figure 6}

Report summary of patient 5 . Major variants reported by the laboratories and VAF concordance with the $\mathrm{CV}$ for the patient 5 are shown. a) Variants reported by the participating laboratories are presented, and information about the variants is presented in the following panels b), c) and d). The variants highlighted in bold should be detected because of their relationship with therapeutics. (G) represents the germline variant. b) Report status for the variants indicated in a) are presented. "1st time" indicates reporting at the first time without information, "2nd time" indicates reporting after the question regarding the existence of the variant, and "not detected" indicates no reporting after the question. c) VAF of the reported variants is presented. Open diamonds and closed circles indicate capture-based and amplicon-based methods, respectively. The vertical bar indicates the ddPCR results, assayed in triplicate. d) CVs (\%) of the VAF in the laboratory reports are shown. 

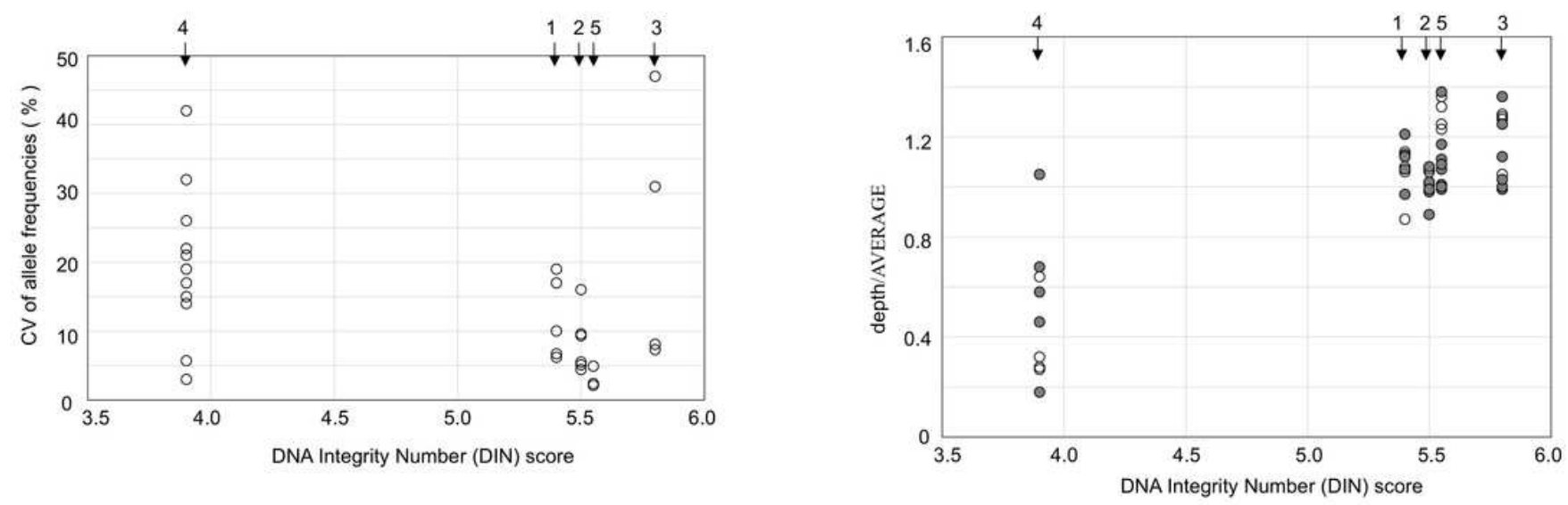

Fig $7 \mathrm{sa}$

\section{Figure 7}

Correlation between DNA quality and NGS results. a) Correlation between DNA quality and CV of allele frequencies for each mutation. Arrows and numbers indicate the number of patient samples. Each circle indicated the $\mathrm{CV}$ of allele frequencies of the detected variants. b) Correlation between DNA quality and depth of sequencing coverage for each laboratory. Arrows and numbers indicate the patient samples. Open and closed circles indicate the capture-based and amplicon-based methods, respectively.

\section{Supplementary Files}

This is a list of supplementary files associated with this preprint. Click to download.

- SupplTable1.pdf

- SupplTable2.pdf

- SupplTable3.pdf

- SupplFig1.pdf

- SupplFig2.pdf

- SupplFig3.pdf 